\title{
Clinical evidence of the efficacy of everolimus and its potential in the treatment of breast cancer
}

\author{
This article was published in the following Dove Press journal: \\ Breast Cancer:Targets and Therapy \\ 16 May 2013 \\ Number of times this article has been viewed
}

\section{Rujuta Saksena \\ Serena TWong}

The Cancer Institute of New Jersey, New Brunswick, NJ, USA
Correspondence: Serena T Wong The Cancer Institute of New Jersey, 195 Little Albany St, New Brunswick, NJ 0890I, USA

Tel +l 7322356249

Email wongse@umdnj.edu

\begin{abstract}
The PI3K/Akt/mTOR (phosphatidylinositol 3-kinase/protein kinase B/mammalian target of rapamycin) pathway regulates several key cellular functions and its dysregulation creates an environment that promotes tumorigenesis as well as resistance to therapy. The mTOR inhibitor everolimus has emerged as a promising agent in the treatment of breast cancer and was recently approved in combination with exemestane for advanced hormone receptorpositive disease after progression on a nonsteroidal aromatase inhibitor. Everolimus may also be effective in combination with cytotoxic and human epidermal growth factor receptor-2directed therapies for the treatment of other subtypes of breast cancer. This paper highlights preclinical and clinical data that have emerged on the role of mTOR inhibition in breast cancer Although generally well tolerated, everolimus carries a unique side effect profile of which both patients and providers should be made aware. Recommendations related to the administration of everolimus in the clinical setting are also discussed.
\end{abstract}

Keywords: everolimus, breast cancer, mTOR inhibition

\section{Introduction}

It is estimated that in 2012 there were approximately 226,870 new cases of invasive breast cancer among women in the United States. An estimated 39,920 breast cancer deaths were expected, making it the second leading cause of cancer death in women. Since 1990, death rates for breast cancer have steadily decreased, reflecting progress in earlier detection and improved treatment. ${ }^{1}$

Treatment strategies for breast cancer vary depending on stage, hormone receptor status, human epidermal growth factor receptor-2 (HER2) status, and other tumor- and patient-specific characteristics. However, one of the major challenges of treatment is overcoming intrinsic and/or acquired drug resistance. Multiple mechanisms of resistance exist, including reduced accumulation of chemotherapy in cancer cells, alterations in drug targets, activation of detoxifying mechanisms, increased repair of drug-induced cellular damage, and alterations in cell signaling, cell cycle control, and apoptosis signaling. ${ }^{2}$ Each one of these mechanisms represents a potential opportunity to develop therapeutic agents in an effort to circumvent resistance.

The field of oncology has witnessed a paradigm shift from a "one size fits all" approach to treatment to a more individualized approach in which a deeper understanding of target oncogenic proteins and pathways has allowed us to develop more rational therapies, resulting in increased efficacy and decreased toxicity.

This paper discusses our understanding of the phosphatidylinositol 3-kinase/ protein kinase $\mathrm{B} / \mathrm{mammalian}$ target of rapamycin $(\mathrm{PI} 3 \mathrm{~K} / \mathrm{Akt} / \mathrm{mTOR}$ ) pathway in 
the pathogenesis of breast cancer as well as the successful development of an inhibitor of this pathway, everolimus, and its role in the treatment of breast cancer.

\section{PI3K/Akt/mTOR pathway}

The PI3K/Akt/mTOR pathway regulates several normal cellular functions, including cellular growth, proliferation, survival, and differentiation. ${ }^{3}$ Dysregulation of this pathway creates an environment that promotes tumorigenesis, and activating mutations are frequently reported in human cancers. ${ }^{4}$

Activation of this pathway can occur through activation or mutation of either PI3K or Akt, overexpression of growth factor receptors such as HER2 or insulin-like growth factor receptor, or through loss of tumor suppressor phosphatase and tensin homolog deleted from chromosome 10 (PTEN) activity. ${ }^{5-7}$

Activated PI3K leads to downstream phosphorylation of Akt, a serine/threonine kinase, which in turn activates other key downstream effectors such as mTOR. mTOR is a serine/ threonine kinase that is a key mediator of cellular proliferation, apoptosis, angiogenesis, and cellular metabolism.

The effects of mTOR are mediated by two distinct multiprotein complexes, mTORC1, which is sensitive to inhibition by rapamycin, and $\mathrm{mTORC} 2$, which is not. ${ }^{8} \mathrm{mTORC} 1$ exerts its effects via phosphorylation of the eukaryotic initiation factor 4E-binding protein 1 (4E-BP1) and the $\mathrm{p} 70$ ribosomal S6 kinase 1 (S6K1), leading to enhanced mRNA translation, cell proliferation, growth, and survival. The function of mTORC2 is incompletely understood but is thought to be related to cytoskeleton organization as well as cell proliferation, survival, and metabolism. ${ }^{8,9}$

Mutations in the PI3K catalytic alpha subunit (PIK3CA) gene and the loss of PTEN, which inhibits the mTOR pathway, are frequently observed in breast cancer. ${ }^{10}$ Several studies have shown that alterations of the PIK3CA gene are observed in approximately $10 \%-40 \%$ of breast cancers. ${ }^{11}$ Activation of the PI3K/Akt signaling pathway in breast cancer cells leads to the development of resistance to therapy, and high Akt and mTOR activity are especially associated with the development of resistance to endocrine therapy. ${ }^{12-14}$ Similar associations have been made between mTOR activation and trastuzumab resistance. ${ }^{15-17}$ These findings provide the rationale for the addition of mTOR inhibition to chemotherapy, endocrine therapy, anti-HER2 therapy, or a combination of these, in an effort to delay or reverse resistance.

\section{mTOR inhibitors}

Rapamycin is a naturally occurring fungicide produced by the bacterium Streptomyces hygroscopicus. It forms a complex with the cytosolic protein FK-binding protein 12 (FKBP12) which blocks the actions of 4E-BP1 and p70 ribosomal S6K, resulting in cell cycle arrest. ${ }^{18}$ Historically, rapamycin has been used as an immunosuppressant in solid organ transplant recipients. In recent years, the use of synthetic rapamycin analogs, or "rapalogs," with more favorable pharmacokinetic properties, has extended to the field of oncology.

Temsirolimus (Torisel ${ }^{\circledR}$, Pfizer, Inc, New York, NY, USA) is US Food and Drug Administration (FDA)-approved for the treatment of renal cell carcinoma; everolimus (Afinitor ${ }^{\circledR}$, Novartis Pharmaceuticals Corporation, East Hanover, NJ, USA) is FDA-approved for the treatment of renal cell carcinoma, pancreatic neuroendocrine tumor, angiomyolipoma, advanced estrogen receptor (ER)+/HER2 breast cancer, and subependymal giant cell astrocytoma in individuals with tuberous sclerosis complex; ridaforolimus is currently in clinical development.

\section{Preclinical data}

Several preclinical studies have demonstrated the efficacy of mTOR inhibition in breast cancer. One of the drivers of the PI3K pathway is ER activation, and activation of both PI3K and Akt may be invoked by crosstalk between epidermal growth factor receptor and ER. Such crosstalk has been associated with estrogen-independent transcriptional activity and estrogen resistance. ${ }^{19-21}$ DeGraffenried et al demonstrated that breast cancer cells with constitutively active Akt proliferate in the absence of exogenous estrogen and develop resistance to the growth inhibitory and proapoptotic effects of tamoxifen both in vitro and in vivo. Cotreatment of these cells with rapamycin derivatives restores the apoptotic responses to tamoxifen. ${ }^{20}$

When everolimus is combined with letrozole or 4-hydroxytamoxifen (an active metabolite of tamoxifen), there is synergistic inhibition of the proliferation of estrogendependent breast cancer cells and increased apoptosis. ${ }^{22,23}$ In another study, coadministration of everolimus with letrozole and fulvestrant reversed Akt-mediated resistance to endocrine therapy and restored responsiveness to endocrine therapy in breast cancer cell lines. ${ }^{24}$ Thus, ample preclinical data are available supporting the potential role of mTOR inhibitors in the treatment of breast cancer, particularly in endocrine-resistant tumors.

\section{Temsirolimus}

A randomized three-arm Phase II study evaluated temsirolimus, also known as CCI-779 or Torisel, in combination with 
letrozole in postmenopausal women with heavily pretreated $\mathrm{ER}+$ metastatic breast cancer (MBC). Patients received letrozole $2.5 \mathrm{mg}$ /day alone or with temsirolimus given orally on a daily or intermittent schedule. The combination showed tolerability and there was a suggestion that progression-free survival (PFS) was longer with the addition of temsirolimus. ${ }^{25}$

A subsequent Phase III study evaluated first-line letrozole $2.5 \mathrm{mg}$ /day plus temsirolimus $30 \mathrm{mg} /$ day ( 5 days every 2 weeks) versus letrozole plus placebo in 1112 patients with aromatase inhibitor (AI)-naïve, hormone receptor-positive advanced breast cancer. However, the study was terminated prematurely after a planned interim analysis showed that the combination was unlikely to achieve the expected level of efficacy. There was no improvement in the primary endpoint of PFS (median, 9 months; hazard ratio (HR), 0.90; 95\% CI, $0.76-1.07 ; P=0.25) .{ }^{26}$ Possible reasons for the failure of the study to meet its primary endpoint include patient selection and/or suboptimal dosing of the drug.

\section{Everolimus}

The rapamycin analog everolimus, also known as RAD 001 or Afinitor, is a highly specific mTOR inhibitor that also carries anti-angiogenic properties. After oral administration, everolimus is absorbed rapidly, with peak concentrations occurring at 1.3-1.8 hours after a single dose. After multiple doses, steady-state concentrations are achieved in approximately 7 days. It has a half-life of 18-35 hours. Everolimus has a predominantly hepatic clearance, and dose adjustment is not needed for renal insufficiency as only $5 \%$ of the drug is excreted in the urine. ${ }^{27}$

Currently, everolimus is FDA-approved for use in advanced renal cell cancer, pancreatic neuroendocrine cancers, and subependymal giant cell astrocytomas. Its most recent FDA approval was granted in July 2012 for treatment of postmenopausal women with advanced hormone receptorpositive, HER2-negative breast cancer in combination with exemestane. This last indication was granted on the basis of study results that will be discussed below.

\section{Everolimus in the treatment of hormone receptor-positive breast cancer}

On the basis of the preclinical data discussed above, a number of studies were conducted evaluating the role of everolimus in the clinical setting.

A Phase I, dose-escalating study evaluated everolimus plus letrozole in 18 postmenopausal patients with stable $\mathrm{MBC}$ or progression after at least 4 months of first- or secondline therapy with letrozole alone. ${ }^{28}$ Six patients received everolimus $5 \mathrm{mg} /$ day, and 12 patients received $10 \mathrm{mg} /$ day. Among these patients, one had a complete response (CR) lasting more than 22 months, and another experienced a $28 \%$ reduction in liver metastases. Both had received everolimus $10 \mathrm{mg} /$ day. There was one dose-limiting toxicity, grade 3 thrombocytopenia, which occurred in a patient assigned to the higher dose. Based on the results of this study, everolimus at a daily dose of $10 \mathrm{mg}$ /day was recommended for subsequent studies.

A Phase II study randomized 270 postmenopausal women with operable ER+ breast cancer to receive 4 months of neoadjuvant treatment with letrozole $2.5 \mathrm{mg}$ /day plus either everolimus $10 \mathrm{mg}$ /day or placebo. The primary endpoint was clinical response by palpation. The response rate (RR) in the everolimus arm was higher than that with letrozole alone (68.1\% versus 59.1\%). An antiproliferative response, defined by a reduction in Ki67 expression at day 15 occurred in 52 of $91(57 \%)$ patients in the everolimus arm and in 25 of $82(30 \%)$ patients in the placebo arm $(P<0.01)$. The authors concluded that everolimus significantly increased letrozole efficacy in the neoadjuvant treatment of ER+ breast cancer. ${ }^{29}$

Sabine et al characterized the effects of preoperative everolimus in primary breast cancer patients through gene expression profiling. Twenty-seven patients with ER+ breast cancer completed 11-14 days of neoadjuvant everolimus $5 \mathrm{mg} /$ day. Patients whose tumors responded with significant reductions in proliferation also had significant decreases in the expression of genes involved in cell cycle and p53 signaling pathways. Overall, everolimus was noted to decrease proliferation, increase apoptosis, and reduce Akt/mTOR signaling in tumors. ${ }^{30}$

The Tamoxifen-RAD001 (TAMRAD) study was a Phase II study of 111 patients with ER+, HER2-negative MBC treated previously with AI therapy. ${ }^{31}$ Patients were randomized to either tamoxifen alone or tamoxifen with everolimus $10 \mathrm{mg} /$ day. The primary outcome was clinical benefit rate (CBR), defined as $\mathrm{CR}+$ partial response $(\mathrm{PR})+$ stable disease (SD) at 6 months. Earlier AI therapy had been administered in either the adjuvant (31\%) or metastatic setting (60\%). Patients were stratified according to primary $(49 \%)$ or secondary $(51 \%)$ hormone resistance. Primary resistance was defined as disease relapse during or within 6 months of stopping adjuvant AI therapy, or disease progression within 6 months of starting AI therapy in the metastatic setting. Secondary resistance was defined as disease relapse greater than 6 months after stopping adjuvant AI therapy or progression after 6 months of AI therapy in the metastatic setting. 
The CBR was $42 \%$ in the tamoxifen-alone group, compared with $61 \%$ in the everolimus plus tamoxifen group (exploratory $P=0.045$ ). The time to progression (TTP) was significantly longer in the combination group (8.6 months versus 4.5 months, HR, $0.54 ; 95 \% \mathrm{CI}, 0.36-0.81$ ). At the time of the last update, there were 31 deaths in the tamoxifenalone group, compared with 16 deaths in the combination group. Median overall survival (OS) had not been reached in the combination group; OS was 32.9 months in the tamoxifen group, yielding a HR for survival of $0.45(95 \%$ CI, $0.24-0.81$, exploratory $P=0.007$ ), favoring the addition of everolimus.

Results from exploratory subgroup analyses suggested that the benefit from the addition of everolimus to tamoxifen may have been restricted to patients with secondary hormone resistance. The CBR among patients with secondary resistance was $74 \%$ with the combination, compared with $48 \%$ with tamoxifen alone; whereas the CBR in patients with primary resistance was only slightly better with the addition of everolimus (46\%) compared with tamoxifen alone (36\%). Similarly, the magnitude of improvement in TTP with the combination was greater in patients with secondary resistance (HR, 0.46; 95\% CI, 0.26-0.83) than that observed in patients with primary resistance (HR, 0.70; 95\% CI, 0.40-1.21). The main toxicities associated with tamoxifen plus everolimus were fatigue (72\% versus 53\% with tamoxifen alone), stomatitis (56\% versus $7 \%$ ), rash ( $44 \%$ versus $7 \%$ ), anorexia ( $43 \%$ versus $18 \%$ ), and diarrhea (39\% versus $11 \%)$.

BOLERO-2 was a randomized Phase III trial that enrolled 724 postmenopausal women with ER+/HER2 locally advanced cancer or MBC that was refractory to letrozole or anastrozole. ${ }^{32}$ Patients were randomized in a 2:1 ratio to receive exemestane $25 \mathrm{mg}$ /day plus everolimus $10 \mathrm{mg} /$ day versus exemestane plus placebo. The primary endpoint was PFS. At the time of interim analysis, the group assigned to everolimus plus exemestane demonstrated a significant improvement in PFS (6.9 months) compared with exemestane alone (2.8 months) (HR, 0.43; 95\% CI, 0.35-0.54; $P<0.001)$. The benefit appeared consistent across all subgroups. Overall survival analysis is not yet mature. There were more grade 3 and 4 toxicities observed with the addition of everolimus, the most common being stomatitis ( $8 \%$ versus $1 \%$ ), anemia ( $6 \%$ versus $<1 \%$ ), dyspnea ( $4 \%$ versus $1 \%$ ), hyperglycemia ( $4 \%$ versus $<1 \%$ ), fatigue $(4 \%$ versus $1 \%$ ) and pneumonitis ( $3 \%$ versus $0 \%$ ). Based on results from this study, in July 2012, the FDA approved the use of everolimus in combination with exemestane for hormone receptor-
positive/HER2 advanced breast cancer in postmenopausal women after progression on a nonsteroidal AI.

\section{Everolimus in the treatment of HER2- overexpressing breast cancer}

HER $2+$ breast cancers, which account for $25 \%-30 \%$ of breast cancers, are biologically aggressive and are associated with altered response to therapy and poor clinical outcomes. ${ }^{33}$ The development of trastuzumab, a humanized monoclonal antibody against HER2, represents a major advance in the treatment of both early-stage and advanced HER2+ breast cancer. ${ }^{34-37}$ Nevertheless, many tumors do not respond to trastuzumab-based therapy, and even among those that do, resistance often develops.

HER2 stimulates cell proliferation and survival through the PI3K/Akt/mTOR pathway, ${ }^{38}$ and activation of this pathway is associated with trastuzumab resistance. ${ }^{15-17}$ Preclinical data have shown that everolimus has synergistic activity with trastuzumab and may overcome trastuzumab resistance. ${ }^{39}$

The efficacy of everolimus in women with HER2+ MBC has been evaluated in several Phase I-II studies. In a Phase Ib study, 33 patients who were pretreated with trastuzumab received everolimus $5 \mathrm{mg}$ daily, $10 \mathrm{mg}$ daily, or $30 \mathrm{mg}$ weekly in combination with weekly trastuzumab and paclitaxel on days 1,8 , and 15 of a 28 -day cycle. ${ }^{40}$ Among the 27 patients with measurable disease, two patients had a $\mathrm{CR}$ and 10 patients had a $\mathrm{PR}$, for an overall response rate (ORR) of 44\%. An additional 13 patients (48\%) had SD. The median PFS was 34 weeks.

In a subsequent Phase II follow-up trial, everolimus $10 \mathrm{mg} /$ day in combination with paclitaxel and trastuzumab in doses similar to the Phase Ib study described above was evaluated in patients who were resistant to taxanes and trastuzumab. Among 48 patients, 19\% had a PR and 62\% had SD. Median PFS was 26 weeks. ${ }^{41}$

In another Phase Ib trial, everolimus was evaluated in patients with HER2+ MBC who had progressed after receiving trastuzumab. ${ }^{42}$ Most patients had received taxanes and anthracyclines previously, and $24 \%$ had been pretreated with lapatinib. Patients received everolimus $5 \mathrm{mg} /$ day, $20 \mathrm{mg} /$ week, or $30 \mathrm{mg} /$ week in combination with weekly trastuzumab and vinorelbine on days 1 and 8 of a 21-day cycle. Among 47 patients in all dosage groups, the ORR was $19 \%$ and the $\mathrm{CBR}(\mathrm{CR}+\mathrm{PR}+\mathrm{SD} \geq 24$ weeks) was $54 \%$. Median PFS was 30.7 weeks for the overall population. In the extension phase of the trial, patients were allowed to continue everolimus, and vinorelbine could be discontinued at the investigator's discretion. Two additional patients 
achieved CR, one achieved PR, and the overall median PFS was 41 weeks.

A post hoc analysis was performed that pooled data from the two previously discussed Phase $\mathrm{I}^{40,42}$ and Phase $\mathrm{II}^{41}$ studies to evaluate the efficacy of everolimus in patients pretreated with lapatinib. ${ }^{43}$ Among 101 patients, the ORR was $21 \%$ for those patients treated with lapatinib compared with $29 \%$ for those not treated with lapatinib. The disease control rate was $88 \%$ and $81 \%$, respectively; overall mean PFS was 29.0 and 36.1 weeks, respectively. These data suggest that everolimus, in combination with trastuzumab and chemotherapy, has antitumor efficacy in patients with HER2+ MBC, regardless of whether they were pretreated with lapatinib.

Patients with HER2+ MBC with progressive disease after previous HER2-targeted therapy were evaluated in two Phase I-II trials; data were combined for analysis. ${ }^{44}$ Everolimus was administered at 5 or $10 \mathrm{mg} /$ day plus a trastuzumab $8 \mathrm{mg} / \mathrm{kg}$ loading dose and then $6 \mathrm{mg} / \mathrm{kg}$ every 3 weeks. Among 47 patients, 15\% had PR and 19\% had SD. The median PFS was 4.1 months. Results from this study suggest that everolimus may have promising activity in the absence of cytotoxic chemotherapy.

The abovementioned trials show encouraging results for everolimus use in patients with HER2+ MBC. Two ongoing Phase III studies, BOLERO-1 and BOLERO-3, will help to further define the role of everolimus in this population. ${ }^{45,46}$ The BOLERO-1 trial is evaluating everolimus in combination with paclitaxel and trastuzumab as first-line therapy, and BOLERO-3 is evaluating everolimus in combination with vinorelbine and trastuzumab in patients with previous taxane therapy and trastuzumab resistance.

\section{Everolimus in the treatment of triple- negative breast cancer (TNBC)}

TNBCs lack the expression of ER, progesterone receptor and HER 2 and comprise about $15 \%$ of all breast cancers. ${ }^{47}$ Such tumors are associated with aggressive behavior and worse survival compared with other subtypes of breast cancer. ${ }^{48}$ Treatment of TNBC remains a challenge, and cytotoxic chemotherapy remains the standard.

A Phase II study was conducted to evaluate the role of mTOR inhibition in combination with chemotherapy in this population. ${ }^{49}$ Fifty patients with early-stage or locally advanced TNBC were randomized to receive neoadjuvant weekly paclitaxel $80 \mathrm{mg} / \mathrm{m}^{2}$ either alone or in combination with everolimus $30 \mathrm{mg} /$ week for 12 weeks followed by 5-fluorouracil, epirubicin, and cyclophosphamide every 3 weeks for four cycles. The 12-week RR by ultrasonography was $48 \%$ when everolimus was administered in combination with paclitaxel compared with $30 \%$ when paclitaxel was used alone $(P=0.15)$. Pathologic $\mathrm{CR}$ rate was not significantly different between the two groups.

Another Phase II study evaluated the combination of everolimus at a dose of $5 \mathrm{mg}$ daily plus carboplatin (area under the curve $[\mathrm{AUC}]=6$ ) given every 3 weeks in patients with metastastic TNBC. ${ }^{50}$ The primary objective was CBR. At the time of reporting, 18 out of a planned total of 25 patients had been enrolled. One patient achieved a CR; four achieved a PR, and two SD lasting greater than 6 months. One of the patients who achieved SD had progressed on singleagent carboplatin at the time of study entry. Dose-limiting thrombocytopenia was an unexpected toxicity requiring amendment of carboplatin dosing to $\mathrm{AUC}=4$.

Due to the small sample sizes of the above studies, no clear conclusions can be drawn at this time. More studies are needed in this population to determine whether any subset exists that may benefit from the addition of everolimus.

\section{Everolimus - toxicities and management}

Safety data from the pivotal BOLERO-2 study showed that the most common adverse events (AEs) with an incidence of $30 \%$ or greater were stomatitis, infections, rash, fatigue, diarrhea, and decreased appetite. Grade 3/4 AEs with an incidence of $2 \%$ or greater included stomatitis, infections, hyperglycemia, fatigue, dyspnea, pneumonitis, and diarrhea. All these AEs occurred with greater frequency in the combination arm. ${ }^{32}$

Noninfectious pneumonitis is a rare but serious AE known to occur with mTOR inhibitors. ${ }^{52}$ It should be considered in patients presenting with cough, dyspnea, hypoxia, or pleural effusions when other causes have been excluded. On computed tomography scans, pneumonitis can present as ground glass or patchy opacities or infiltrates. It appears to be immunologically mediated, with biopsies showing organizing pneumonia, granulomatous inflammation, and lymphocytic infiltration or vasculitis. Management of symptomatic pneumonitis involves cessation of therapy and the use of corticosteroids if severe. Most cases have been reversible upon drug discontinuation. A pooled analysis of five studies evaluating everolimus in breast cancer patients reported variability in the incidence of noninfectious pneumonitis. In four of the studies, the incidence was approximately $3 \%$; however, the incidence was $35 \%$ in the fifth study. ${ }^{51}$

The immunosuppressive properties of everolimus may predispose patients to opportunistic infections and reactivation of previous infections including hepatitis B. Pneumonia, 


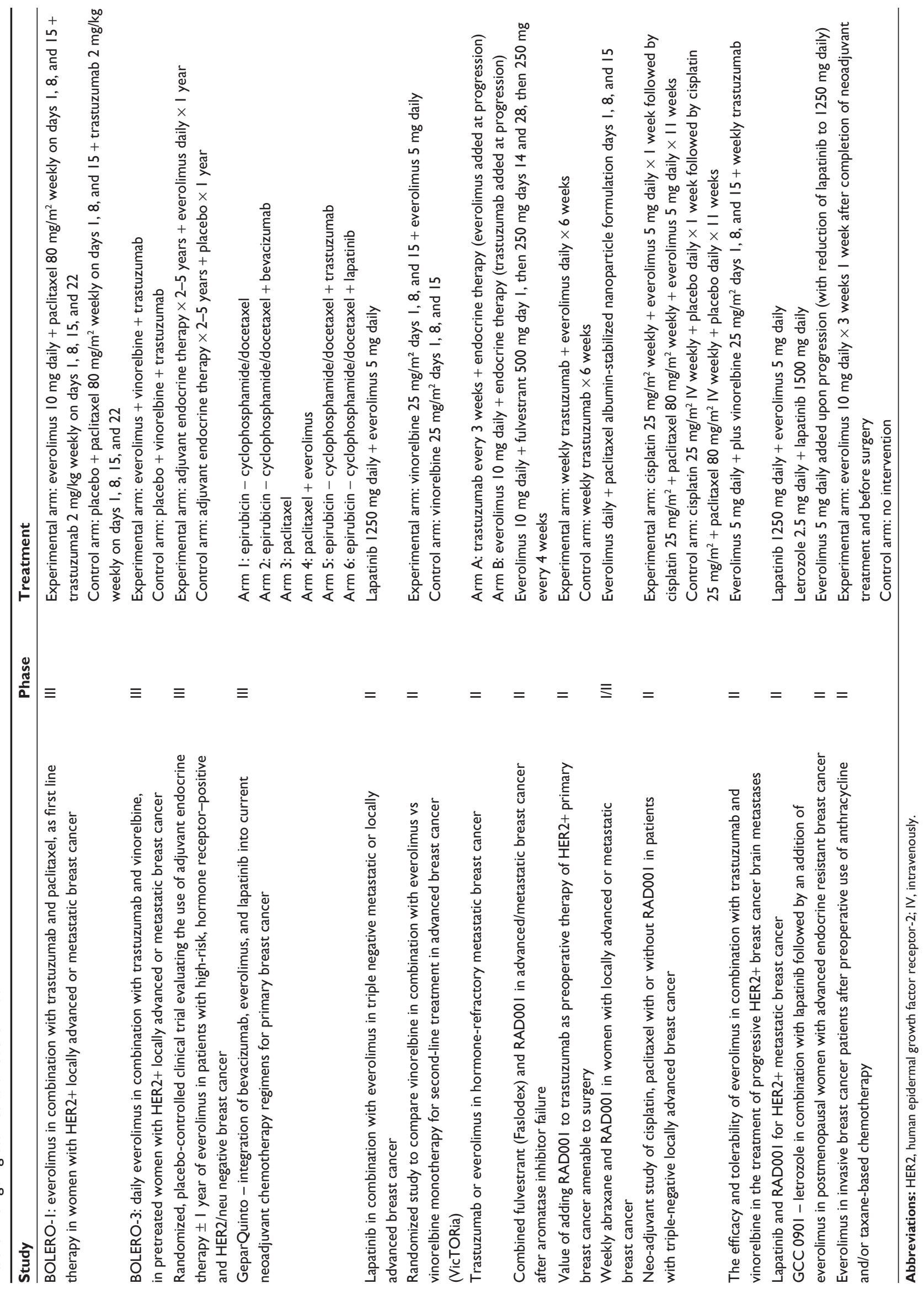


mycobacterial infections, other bacterial infections, and invasive fungal infections have been noted in those treated with everolimus. ${ }^{52}$

Everolimus is also associated with metabolic derangements, notably hyperglycemia, hypercholesterolemia, and hypertriglyceridemia. ${ }^{52}$ Optimal glycemic and lipid control should be achieved before starting everolimus, and serum glucose and lipid levels should be checked at baseline and periodically during treatment. These abnormalities can be treated using standard guidelines for diabetes and hyperlipidemia.

\section{Everolimus - drug interactions}

Everolimus is a substrate of CYP3A4 and P-glycoprotein. Coadministration with ketoconazole, a strong CYP3A4 inhibitor, results in increased everolimus blood concentrations, with resultant maximum concentration $\left(\mathrm{C}_{\max }\right)$ and $\mathrm{AUC}$ 3.9-fold and 15-fold higher, respectively. It is recommended that concurrent administration of strong CYP3A4 inhibitors be avoided. If coadministration with a moderate inhibitor is necessary, it is suggested that the dose of everolimus be reduced to avoid excess toxicity. Conversely, coadministration with the strong CYP3A4 inducer rifampin decreases $\mathrm{C}_{\max }$ and $\mathrm{AUC}$ of everolimus by $58 \%$ and $63 \%$, respectively. If concurrent use of a strong inducer is necessary, it is suggested that the dose of everolimus be increased. ${ }^{52}$

\section{Predictive biomarkers}

In this era of targeted therapy it is crucial to be able to identify subsets of patients who are either likely or unlikely to respond to a given drug. Such identification would maximize efficacy and minimize unnecessary toxicity. Currently, no reliable biomarkers exist to predict response to treatment with everolimus, although some molecular patterns are emerging as potential predictors of sensitivity and/or resistance.

Di Nicolantonio et al performed a series of elegant experiments to assess the sensitivity to everolimus of various cancer cell lines harboring mutations in PIK3CA or PTEN. ${ }^{53}$ They found that the tumors could be divided into two groups based on response to everolimus: those resistant to treatment harbored both a mutation in the PI3K pathway as well as a mutation in either $K R A S$ or $B R A F$. On the other hand, those that were sensitive to everolimus had only a mutation in the PI3K pathway. These results suggest that treatment with an mTOR inhibitor may be ineffective against $K R A S$ or $B R A F$ mutant tumors despite the presence of $\mathrm{PI} 3 \mathrm{~K} / \mathrm{AKT} / \mathrm{mTOR}$ pathway activation.
This hypothesis was tested by Janku et al in a study evaluating response in 25 patients with breast or gynecologic tumors harboring the PIK3CA mutation, 23 of whom were treated on a protocol that included a PI3K/AKT/mTOR pathway inhibitor. ${ }^{54}$ Two (9\%) of the 23 patients had SD for more than 6 months, and seven patients (30\%) had a PR. Seven patients with $P I K 3 C A$ mutations had coexisting mutations in $K R A S, N R A S$, or $B R A F$, and two of these patients (ovarian cancer) achieved a response. Although the numbers are small, these findings suggest that not all patients with simultaneous mutations in both pathways demonstrate resistance.

Chen et al found that expression of the cell cycle regulator p27 correlated with the anticancer activity of rapamycin and temsirolimus in breast cancer cells in vitro and in vivo. Cells expressing high levels of p27 were sensitive to treatment, whereas those with low expression demonstrated resistance. Moreover, they observed consistently that downregulation of p 27 by silencing RNA rendered cells with normally high levels of expression resistant to treatment. They propose that p27 expression levels might serve as a predictive biomarker for patient selection for rapalog-based therapy. ${ }^{55}$

\section{Conclusion}

The mTOR pathway is pivotal to the pathogenesis of many cancers, including breast cancer. Dysregulation of this pathway is associated with resistance both to endocrine and to HER2-directed therapies. The mTOR inhibitor everolimus has emerged as a promising agent in the treatment of several cancers and is now approved in combination with exemestane in postmenopausal hormone receptor-positive advanced breast cancer.

Everolimus carries a unique side-effect profile, which clinicians and patients should be aware of. With proper pretreatment planning and careful monitoring, treatmentrelated toxicities are generally manageable and the drug well tolerated.

Studies are ongoing to further define the role of everolimus in various subtypes and stages of breast cancer (see Table 1). Identification of biomarkers capable of predicting sensitivity or resistance to mTOR inhibition in breast cancer remains an unmet need. As our understanding of the molecular profiles of tumors improves, we will be able to develop increasingly refined targeted agents as well as appropriate selection criteria that will lead to improved outcomes with minimal toxicities.

\section{Disclosure}

The authors disclose no potential conflicts of interest. 


\section{References}

1. American Cancer Society. Cancer Facts and Figures 2012. Atlanta: American Cancer Society; 2012.

2. Germano S, O'Driscoll L. Breast cancer: understanding sensitivity and resistance to chemotherapy and targeted therapies to aid in personalised medicine. Curr Cancer Drug Targets. 2009;9(3):398-418.

3. Vivanco I, Sawyers CL. The phosphatidylinositol 3-kinase AKT pathway in human cancer. Nat Rev Cance. 2002;2(7):489-501.

4. Wood LD, Parsons DW, Jones S, et al. The genomic landscapes of human breast and colorectal cancers. Science. 2007;318(5853):1108-1113.

5. Cui X, Zhang P, Deng W, et al. Insulin-like growth factor-I inhibits progesterone receptor expression in breast cancer cells via the phosphatidylinositol 3-kinase/Akt/mammalian target of rapamycin pathway: progesterone receptor as a potential indicator of growth factor activity in breast cancer. Mol Endocrinol. 2003;17(4):575-588.

6. Zhou BP, Hu MC, Miller SA, et al. HER-2/neu blocks tumor necrosis factor-induced apoptosis via the Akt/NF-kappaB pathway. J Biol Chem. 2000;275(11):8027-8031.

7. Stemke-Hale K, Gonzalez-Angulo AM, Lluch A, et al. An integrative genomic and proteomic analysis of PIK3CA, PTEN, and AKT mutations in breast cancer. Cancer Res. 2008;68(15):6084-6091.

8. Wullschleger S, Loewith R, Hall MN. TOR signaling in growth and metabolism. Cell. 2006;124(3):471-484.

9. Guertin DA, Sabatini DM. Defining the role of mTOR in cancer. Cancer Cell. 2007;12(1):9-22.

10. Perez-Tenorio G, Alkhori L, Olsson B, et al. PIK3CA mutations and PTEN loss correlate with similar prognostic factors and are not mutually exclusive in breast cancer. Clin Cancer Res. 2007;13(12):3577-3584.

11. Karakas B, Bachman KE, Park BH. Mutation of the PIK3CA oncogene in human cancers. Br J Cancer. 2006;94(4):455-459.

12. deGraffenried LA, Friedrichs WE, Russell DH, et al. Inhibition of mTOR activity restores tamoxifen response in breast cancer cells with aberrant Akt Activity. Clin Cancer Res. 2004;10(23):8059-8067.

13. Musgrove EA, Sutherland RL. Biological determinants of endocrine resistance in breast cancer. Nat Rev Cancer. 2009;9(9):631-643.

14. Chollet $\mathrm{P}$, Abrial $\mathrm{C}$, Tacca $\mathrm{O}$, et al. Mammalian target of rapamycin inhibitors in combination with letrozole in breast cancer. Clin Breast Cancer. 2006;7(4):336-338.

15. Nagata Y, Lan KH, Zhou X, et al. PTEN activation contributes to tumor inhibition by trastuzumab, and loss of PTEN predicts trastuzumab resistance in patients. Cancer Cell. 2004;6(2):117-127.

16. Berns K, Horlings HM, Hennessy BT, et al. A functional genetic approach identifies the PI3K pathway as a major determinant of trastuzumab resistance in breast cancer. Cancer Cell. 2007;12(4): 395-402.

17. Kataoka Y, Mukohara T, Shimada H, Saijo N, Hirai M, Minami H. Association between gain-of-function mutations in PIK3CA and resistance to HER2-targeted agents in HER2-amplified breast cancer cell lines. Ann Oncol. 2010;21(2):255-262.

18. Bjornsti MA, Houghton PJ. The TOR pathway: a target for cancer therapy. Nat Rev Cancer. 2004;4(5):335-348.

19. Campbell RA, Bhat-Nakshatri P, Patel NM, Constantinidou D, Ali S, Nakshatri H. Phosphatidylinositol 3-kinase/AKT-mediated activation of estrogen receptor alpha: a new model for anti-estrogen resistance. J Biol Chem. 2001;276(13):9817-9824.

20. DeGraffenried L, Friedrichs W, Fulcher L. The mTOR inhibitor CCI-779 restores tamoxifen response in breast cancer cells with high Akt activity. Proceedings of the 14th NCI-EORTC-AACR Symposium on Molecular Targets and Cancer Therapeutics. Eur J Cancer. 2002:S158.

21. Smith CL. Cross-talk between peptide growth factor and estrogen receptor signaling pathways. Biol Reprod. 1998;58(3):627-632.

22. Boulay A, Rudloff J, Ye J, et al. Dual inhibition of mTOR and estrogen receptor signaling in vitro induces cell death in models of breast cancer. Clin Cancer Res. 2005;11(14):5319-5328.

23. Treeck O, Wackwitz B, Haus U, Ortmann O. Effects of a combined treatment with mTOR inhibitor RAD001 and tamoxifen in vitro on growth and apoptosis of human cancer cells. Gynecol Oncol. 2006;102(2): 292-299.
24. Beeram M, Tan QT, Tekmal RR, Russell D, Middleton A, DeGraffenried LA. Akt-induced endocrine therapy resistance is reversed by inhibition of mTOR signaling. Ann Oncol. 2007;18(8): 1323-1328.

25. Carpenter JT, Roché H, Campone M, et al. Randomized 3-arm, Phase 2 study of temsirolimus (CCI-779) in combination with letrozole in postmenopausal women with locally advanced or metastatic breast cancer. J Clin Oncol. 2005 ASCO Annual Meeting Proceedings. Vol 23(Suppl 16), Part I of II (June 1 Suppl), 2005:564.

26. Wolff AC, Lazar AA, Bondarenko I, et al. Randomized Phase III placebo-controlled trial of letrozole plus oral temsirolimus as first-line endocrine therapy in postmenopausal women with locally advanced or metastatic breast cancer. J Clin Oncol. 2013;31(2):195-202.

27. Kirchner GI, Meier-Wiedenbach I, Manns MP. Clinical pharmacokinetics of everolimus. Clin Pharmacokinet. 2004;43(2):83-95.

28. Awada A, Cardoso F, Fontaine C, et al. The oral mTOR inhibitor RAD001 (everolimus) in combination with letrozole in patients with advanced breast cancer: results of a phase I study with pharmacokinetics. Eur J Cancer. 2008;44(1):84-91.

29. Baselga J, Semiglazov V, van Dam P, et al. Phase II randomized study of neoadjuvant everolimus plus letrozole compared with placebo plus letrozole in patients with estrogen receptor-positive breast cancer. J Clin Oncol. 2009;27(16):2630-2637.

30. Sabine VS, Sims AH, Macaskill EJ, et al. Gene expression profiling of response to mTOR inhibitor everolimus in pre-operatively treated post-menopausal women with oestrogen receptor-positive breast cancer. Breast Cancer Res Treat. 2010;122(2):419-428.

31. Bachelot T, Bourgier C, Cropet C, et al. Randomized Phase II trial of everolimus in combination with tamoxifen in patients with hormone receptor-positive, human epidermal growth factor receptor 2-negative metastatic breast cancer with prior exposure to aromatase inhibitors: a GINECO study. J Clin Oncol. 2012;30(22):2718-2724.

32. Baselga J, Campone M, Piccart M, et al. Everolimus in postmenopausal hormone-receptor-positive advanced breast cancer. $N$ Engl J Med. 2012;366(6):520-529.

33. Slamon DJ, Clark GM, Wong SG, Levin WJ, Ullrich A, McGuire WL. Human breast cancer: correlation of relapse and survival with amplification of the HER-2/neu oncogene. Science. 1987;235(4785): 177-182.

34. Slamon D, Eiermann W, Robert N, et al. Adjuvant trastuzumab in HER2positive breast cancer. N Engl J Med. 2011;365(14):1273-1283.

35. Slamon DJ, Leyland-Jones B, Shak S, et al. Use of chemotherapy plus a monoclonal antibody against HER2 for metastatic breast cancer that overexpresses HER2. N Engl J Med. 2001;344(11):783-792.

36. Romond EH, Perez EA, Bryant J, et al. Trastuzumab plus adjuvant chemotherapy for operable HER2-positive breast cancer. NEngl J Med. 2005;353(16):1673-1684.

37. Piccart-Gebhart MJ, Procter M, Leyland-Jones B, et al. Trastuzumab after adjuvant chemotherapy in HER2-positive breast cancer. $N$ Engl J Med. 2005;353(16):1659-1672.

38. Harari D, Yarden Y. Molecular mechanisms underlying ErbB2/HER2 action in breast cancer. Oncogene. 2000;19(53):6102-6114.

39. Lu CH, Wyszomierski SL, Tseng LM, et al. Preclinical testing of clinically applicable strategies for overcoming trastuzumab resistance caused by PTEN deficiency. Clin Cancer Res. 2007;13(19):5883-5888.

40. Andre F, Campone M, O'Regan R, et al. Phase I study of everolimus plus weekly paclitaxel and trastuzumab in patients with metastatic breast cancer pretreated with trastuzumab. J Clin Oncol. 2010;28(34): 5110-5115.

41. Dalenc F, Campone M, Hupperets $P$, et al. Everolimus in combination with weekly paclitaxel and trastuzumab in patients (pts) with HER-2overexpressing metastatic breast cancer (MBC) with prior resistance to trastuzumab and taxanes: a multicenter phase II clinical trial. Proceedings of the American Society of Clinical Oncology Annual Meeting; June 4-8, 2010; Chicago, IL, USA.

42. Jerusalem G, Fasolo A, Dieras V, et al. Phase I trial of oral mTOR inhibitor everolimus in combination with trastuzumab and vinorelbine in pre-treated patients with HER2-overexpressing metastatic breast cancer. Breast Cancer Res Treat. 2011;125(2):447-455. 
43. Campone M, Gianni L, Massacesi C, et al. Trastuzumab and everolimus (RAD001) containing regimens are safe and active when reintroduced in patients with HER2-overexpressing metastatic breast cancer (MBC) pre-treated with lapatinib [abstract]. Eur J Cancer Suppl. 2010;8:186.

44. Morrow PK, Wulf GM, Ensor J, et al. Phase I/II study of trastuzumab in combination with everolimus (RAD001) in patients with HER2overexpressing metastatic breast cancer who progressed on trastuzumabbased therapy. J Clin Oncol. 2011;29(23):3126-3132.

45. Everolimus in Combination With Trastuzumab and Paclitaxel in the Treatment of HER2 Positive Locally Advanced or Metastatic Breast Cancer (BOLERO-1). Available from http://clinicaltrials.gov/ct2/show/ NCT00876395. Accessed March 21, 2013.

46. Daily Everolimus in Combination With Trastuzumab and Vinorelbine in HER2/Neu Positive Women With Locally Advanced or Metastatic Breast Cancer (BOLERO-3). Available from http://clinicaltrials.gov/ ct2/show/NCT01007942. Accessed March 21, 2013.

47. Irvin WJ Jr, Carey LA. What is triple-negative breast cancer? Eur $J$ Cancer. 2008;44(18):2799-2805.

48. Dent R, Trudeau M, Pritchard KI, et al. Triple-negative breast cancer: clinical features and patterns of recurrence. Clin Cancer Res. 2007; 13(15 Pt 1):4429-4434.

49. Gonzalez-Angulo A, Green M, Murray J, et al. Open label randomized clinical trial of standard neoadjuvant chemotherapy with paclitaxel followed by FEC (T-FEC) vs the combination of paclitaxel and RAD001 followed by FEC (TR-FEC) in women with triple receptor-negative breast cancer (TNBC). Presented at the American Society of Clinical Oncology Annual Meeting; June 3-7, 2011; Chicago, IL, USA.
50. Singh J, Stein S, Volm M, et al. Phase II trial of RAD001 plus carboplatin in patients with triple-negative metastatic breast cancer. J Clin Oncol. 2012;30(Suppl 15):e11529.

51. Jerusalem G, Ellard S, Fasolo A. Non-infectious pneumonitis in breast cancer patients treated with Everolimus containing therapy: analysis of five studies. Cancer Res. 2009;69(supp 24):1115.

52. Afinitor ${ }^{\circledR}$ (everolimus) tablets for oral administration [package insert]. East Hanover, NJ: Novartis Pharmaceuticals Corporation.

53. Di Nicolantonio F, Arena S, Tabernero J, Grosso S. Deregulation of the PI3K and KRAS signaling pathways in human cancer cells determines their response to everolimus. J Clin Invest. 2010;120(8): 2858-2866.

54. Janku F, Wheler JJ, Westin SN, et al. PI3K/AKT/mTOR inhibitors in patients with breast and gynecologic malignancies harboring PIK3CA mutations. J Clin Oncol. 2012;30(8):777-782.

55. Chen G, Yang N, Wang X, et al. Identification of $\mathrm{p} 27 / \mathrm{KIP} 1$ expression level as a candidate biomarker of response to rapalogs therapy in human cancer. J Mol Med (Berl). 2010;88(9):941-952.
Breast Cancer: Targets and Therapy

\section{Publish your work in this journal}

Breast Cancer: Targets and Therapy is an international, peerreviewed open access journal focusing on breast cancer research, identification of therapeutic targets and the optimal use of preventative and integrated treatment interventions to achieve improved outcomes, enhanced survival and quality of life for the cancer patient.

\section{Dovepress}

View the full aims and scopes of this journal here. The manuscript management system is completely online and includes a very quick and fair peer-review system, which is all easy to use. Visit http:// www.dovepress.com/testimonials.php to read real quotes from published authors. 